\title{
A Review of Neuroimaging in Rare Neurodegenerative Diseases
}

\author{
Michał Sobański ${ }^{a}$ Anna Zacharzewska-Gondek ${ }^{\mathrm{a}}$ Marta Waliszewska-Prosół ${ }^{\mathrm{b}}$ \\ Marek Jan Sąsiadek ${ }^{a}$ Anna Zimny ${ }^{a}$ Joanna Bladowska ${ }^{a}$ \\ aDepartment of General and Interventional Radiology and Neuroradiology, Wroclaw Medical University, \\ Wroclaw, Poland; 'Department of Neurology, Wroclaw Medical University, Wroclaw, Poland
}

\section{Keywords}

Conventional magnetic resonance imaging •

Neurodegenerative diseases - Differential diagnosis ·

Atypical parkinsonian syndromes · Neuroimaging

\begin{abstract}
Purpose: Due to the variety of clinical symptoms that occur in rare neurodegenerative diseases and difficulties in the correct diagnosis, there is a need to learn their characteristic imaging findings by using conventional MRI. That knowledge helps to determine the appropriate differential diagnosis and avoid misdiagnosis. The aim of this review is to present the typical neuroimaging signs of the selected neurodegenerative disorders and to create a practical approach to imaging findings useful in everyday clinical practice. Images: Images of progressive supranuclear palsy (PSP), multiple system atrophy (MSA), corticobasal degeneration (CBD), Creutzfeldt-Jakob disease (CJD), Wilson's disease (WD), and cerebral autosomal dominant arteriopathy with subcortical infarcts and leukoencephalopathy (CADASIL) are provided to visualize and distinguish the typical features of those diseases and therefore to assist neurologists and neuroradiologists in decision-making process. Conclusions: It is
\end{abstract}

important to know the characteristic MRI features of rare neurodegenerative diseases and to use them in everyday clinical practice. MRI is a valuable tool when considering the initial diagnosis because it is proven to be very useful in the differentiation of more advanced stages of the rare neurodegenerative diseases but also from other neurodegenerative disorders.

(c) 2021 S. Karger AG, Basel

\section{Introduction and Aims}

Neurodegenerative diseases are a very common and growing cause of morbidity and mortality worldwide, especially in the elderly group. Presently, known neurodegenerative disorders are heterogeneous in their clinical manifestations as well as their underlying pathophysiology, although their features are often similar and may overlap $[1,2]$.

Neurodegenerative diseases are common cause of cognitive impairment containing a diverse group of entities such as Alzheimer's disease, Parkinson's disease (PD), progressive supranuclear palsy (PSP), multiple system atrophy (MSA), corticobasal degeneration (CBD), demen-

Anna Zacharzewska-Gondek

Department of General and Interventional Radiology and Neuroradiology Wroclaw Medical University

Borowska 213, PL-50-556 Wroclaw (Poland)

aniazacha@gazeta.pl 
Fig. 1. a Hummingbird sign or silhouette penguin sign in midsagittal T2-weighted image typical of PSP. Atrophy of midbrain results in reduction of its anterio-posterior dimension, which creates head of hummingbird. Pons as a body completes the whole sign. b Mickey mouse sign or morning glory sign on axial T2-weighted imagemidbrain atrophy results in the concave lateral margins of the midbrain in a different patient with PSP. PSP, progressive supranuclear palsy.
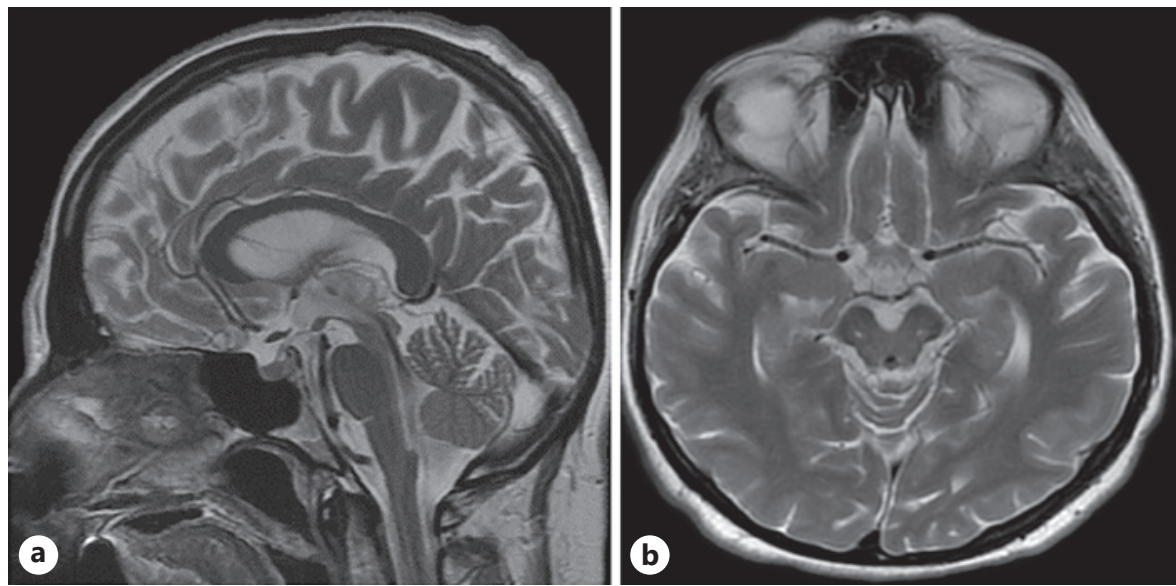

tia with Lewy bodies, frontotemporal dementia and its variants, Huntington's disease (HD), Creutzfeldt-Jakob disease (CJD), Wilson's disease (WD), and cerebral autosomal dominant arteriopathy with subcortical infarcts and leukoencephalopathy (CADASIL). PSP, MSA, CBD, and dementia with Lewy bodies are called atypical parkinsonian syndromes, up to $20 \%$ of parkinsonian spectrum [2]. Each of these diseases has a varied epidemiology, neuropathology, clinical symptomatology, neuroimaging features, and, consequently, management. Correct diagnosis is of key importance in this group of diseases because it allows a more reliable prognosis and most often leads to specific treatment and management of the patients. The objective of this pictorial review is to present the typical neuroimaging findings of the selected rare neurodegenerative disorders and to provide a practical approach to imaging findings useful in everyday clinical practice.

\section{Progressive Supranuclear Palsy}

PSP is characterized by the presence of pathological tau protein [3]. At the neurotransmitter level, dopaminergic nigrostriatal pathway and cholinergic transmission in the brain are disrupted [4]. The prevalence is 5-6 per 100,000 in community-based studies. The mean age of onset is 63 years, and it rarely, if ever, occurs before the age of 40 . The median survival after onset of symptoms is approximately 6 and 8 years. Importantly, only 1.5 per 100,000 are accurately diagnosed $[5,6]$, and consequently approximately 3 of 4 patients are misdiagnosed or not diagnosed at all. In the classic type, PSP is an akineticrigid syndrome with frontal lobe disorder. Balance disor- ders, postural instability and falls, and eye movement abnormalities (a vertical supranuclear gaze palsy and slowed vertical saccades) are commonly encountered in this disease. Parkinsonian features are common, such as: hypomimia, bradykinesia, and sitting "en bloc." Accompanying features include symmetric akinesia or rigidity, dysphagia and dysarthria, retrocollis, and a poor response to dopamine treatment. Cognitive and behavioural changes and depression and sleep disturbances are frequent $[2,7$, 8].

Many diagnostic tests have been found which are useful to distinguish PSP from other conditions with similar symptoms. Differentiation PSP from any other atypical parkinsonian syndromes at early stage using MRI has not been proven an effective method, but MRI is helpful in the more advanced stages. In the clinical practice, diagnosis is based on patient history, neurological examination, and MRI [7]. Conventional MRI may demonstrate abnormalities such as midbrain atrophy, secondary 3rd and 4 th ventricle dilatation, temporal and frontal atrophy, and periaqueductal T2-weighted (T2W) hyperintensities. Midbrain atrophy results in hummingbird sign or penguin-silhouette sign in the midsagittal plane (Fig. 1a) and Mickey mouse sign or morning glory sign in the axial plane (Fig. 1b) [8]. The correct way to demonstrate morning glory sign is on the axial section of midbrain at mammillary body level [9]. However, precise sagittal acquisition is easier to perform and more reliable in detecting the penguin-silhouette sign and, hence, is superior to the morning glory sign in the diagnosis of PSP [9-11]. Another useful tool is a measurement of midbrain major axis and midbrain to pons ratio based on major axes shown on Fig. 2b. Major axis of midbrain measuring $<9.35 \mathrm{~mm}$ and calculated midbrain to pons ratio $<0.52$ 

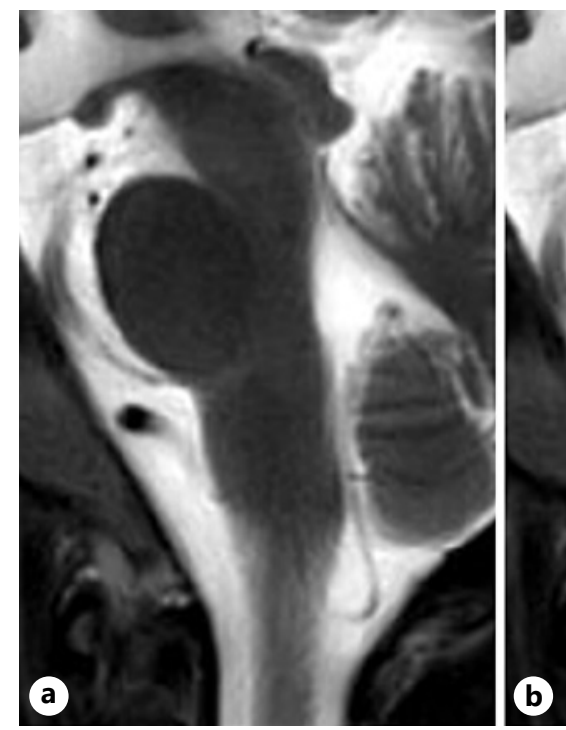

Fig. 2. a-c Midsagittal T2-weighted image of midbrain and pons. b The regions of interest for midbrain and pons are covered by ellipses. Region for midbrain excludes the collicular plate to maximize the chance of detecting atrophy. Region for pons does not include distinctly differentiating pontine tegmentum. Two lines were drawn to establish major axes. Firstly, the maximal axis in oblique superior-inferior was determined (thin line). Secondly, maximal measurement perpendicular to major axis was taken (thick lines) to calculate midbrain to pons ratio. c A scheme pre- senting method of calculating the midbrain to pons ratio on magnified midsagittal T2-weighted image. Two straight lines were imposed: first line was drawn through superior pontine notch and inferior edge of quadrigeminal plate and second parallel line pass through inferior pontine notch. Area of midbrain traced around line 1 and the shape of midbrain tegmentum. Area of pons is the area between horizontal lines 1 and 2 and posterior and anterior borders of pons.
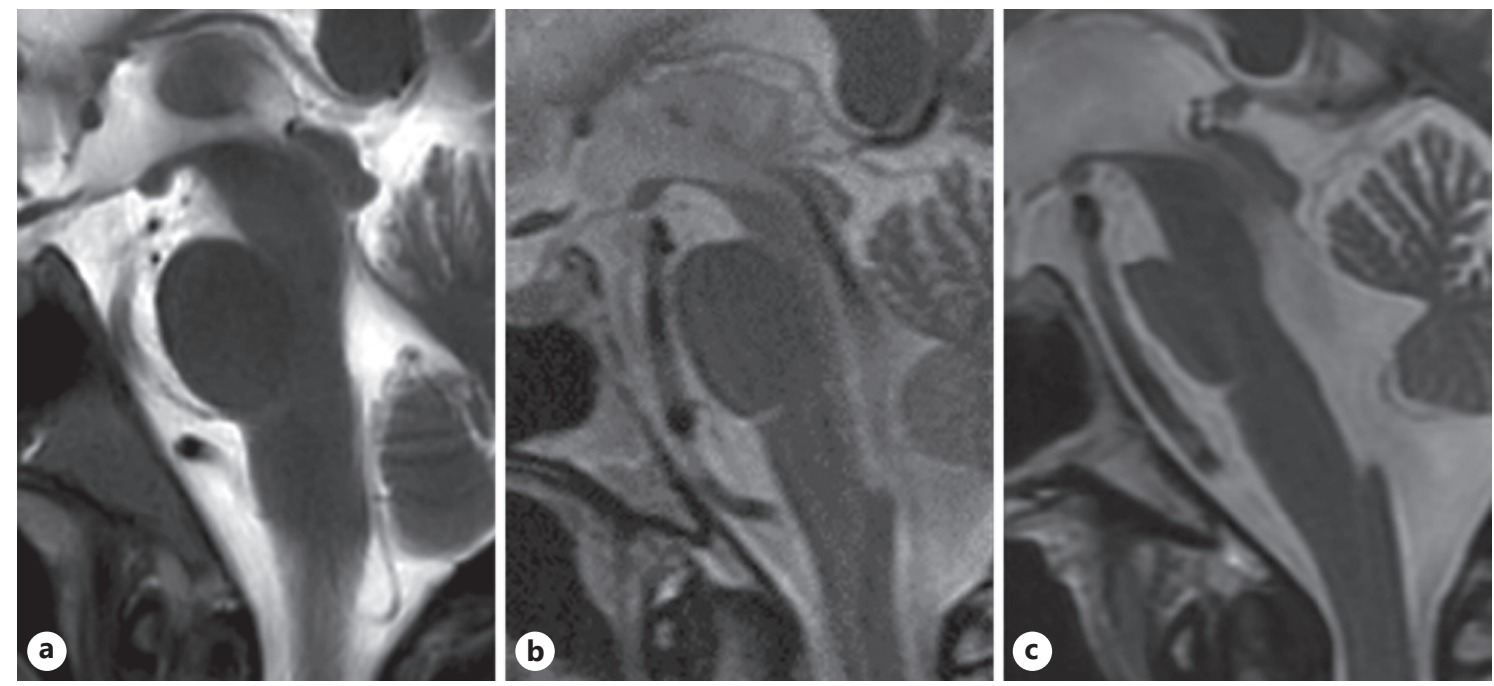

Fig. 3. Midsagittal T2-weighted images of midbrain and pons in 3 different patients. a A healthy patient does not show any visible changes. b A patient with PSP demonstrates notable atrophy of midbrain tegmentum. c A patient with MSA of the cerebellar type reveals pontine atrophy. PSP, progressive supranuclear palsy; MSA, multiple system atrophy. 
Fig. 4. A patient with MSA-P type. Axial T2-weighted (a) and FLAIR images show atrophy and hypointensity of putamen (b), also "slit-like" marginal hyperintensity (arrows). MSA-P, multiple system atrophyparkinsonian type.
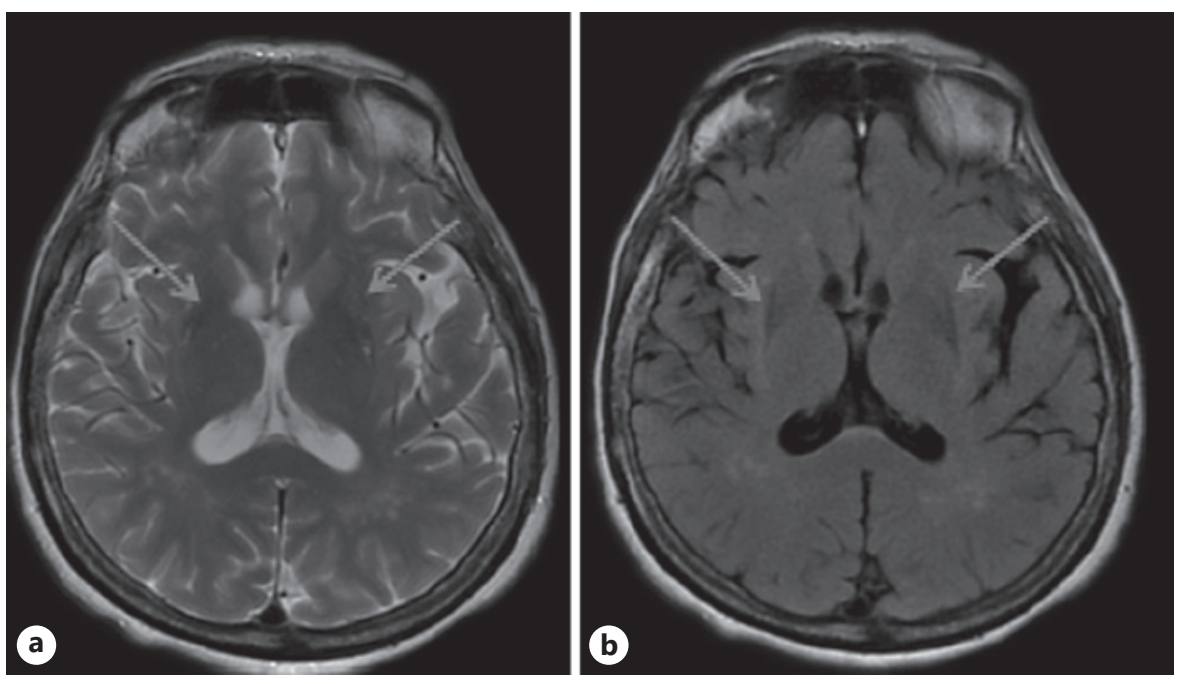

has $100 \%$ specificity for diagnosing PSP. An alternative method to calculate midbrain to pons ratio is to divide midbrain area to pons area measured in the midsagittal plane. Patients with PSP have significantly smaller ratio (0.124) than patients with PD (0.208) or MSA-P (0.266) or the control subjects (0.237) (Fig. 2c) [12], and this measurement may differentiate PSP from PD, MSA, and healthy brain (Fig. 3) [12]. The measurement of a diameter of the midbrain on the axial section is less reliable due to variability of plane orientation, and nevertheless the value of $<14 \mathrm{~mm}$ is very suggestive of PSP $[13,14]$.

\section{Multiple System Atrophy}

MSA is a disorder with predominantly parkinsonian (MSA-P) or cerebellar (MSA-C) signs with accompanying symptoms of the failure of autonomic system [15]. Misfolded $\alpha$-synuclein is main pathological protein in glia, which causes neuroinflammation and destruction of neurons. Characterization and severity of symptoms depend on the location where inflammation occurs. Striatonigral degeneration corresponds with parkinsonian symptoms, olivopontocerebellar atrophy with cerebellar syndrome, and degeneration of autonomic nuclei in the brain stem and spinal cord results in multidomain autonomic failure [16]. The prevalence in age-group 50-99 years for MSA was estimated to be 3.0 per 100,000. Median age of first symptom onset was approximately 66 years and survival time 8.5 years [17]. Parkinsonism, with rigidity, postural instability, slowness of movements, gait disability, tendency to fall, and poor levodopa-response is characteristic for MSA-P. The motor findings are rarely asymmetrical, and rest tremor is rare. Cerebellar ataxia, uncoordinated limb movements, tremor, and nystagmus predominate in MSA-C. In advanced stages occur dysphonia, dysphagia, dystonia, falls, drooling, and pain. An autonomic failure, in particular urogenital and cardiovascular disorders, is frequent early features but is not specific for MSA. However, in a certain group of patients, autonomic dysfunction may be the only presenting feature. Other symptoms' characteristic of this disease include constipation, hypohidrosis, oculomotor dysfunctions, excessive daytime sleepiness, restless legs syndrome, respiratory disturbances (nocturnal inspiratory stridor and sleep apnea) [15-17]. The diagnosis of definite MSA requires neuropathological confirmation. The criteria for possible MSA consist of clinical and neuroimaging features [18].

The most distinctive feature of MSA-P on conventional MRI is the putaminal atrophy, with the hypointensity on T2-weighted images and slit-like marginal hyperintensity (Fig. 4). Hypointense putaminal signal on T2weighted gradient-echo (GE) images and particularly on susceptibility-weighted images (SWI) has been reported as more characteristic for MSA-P than on T2-weighted fast spin echo [19]. Low signal on T2-weighted images, T2*-weighted images and SWI are the results of high accumulation of iron (Fig. 5) [20,21]. Hyperintense slit-like margins on the lateral margin of the putamen are caused by microgliosis and astrogliosis [21]. The cruciform signal hyperintensity on T2-weighted images in the central part of pons (called the hot cross bun sign) and high-signal intensity areas in the middle cerebellar peduncles re- 


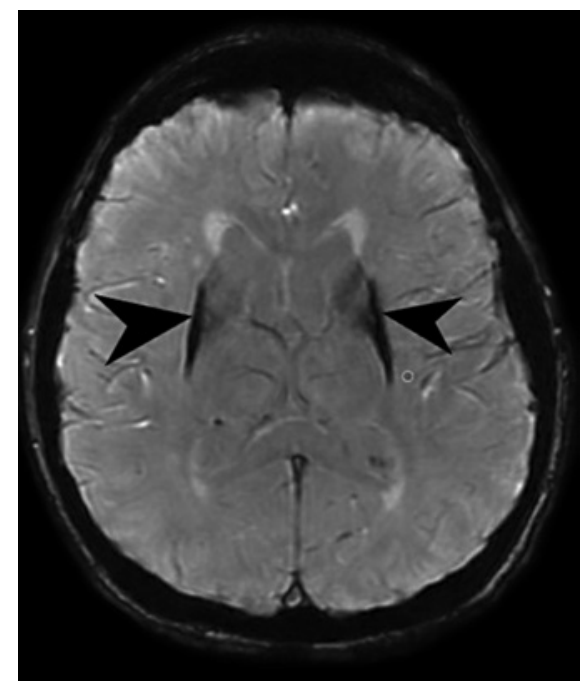

Fig. 5. Axial SWI image showing low signal of both putamina (arrowheads) - a characteristic feature for MSA-P type. MSA-P, multiple system atrophy-parkinsonian type.
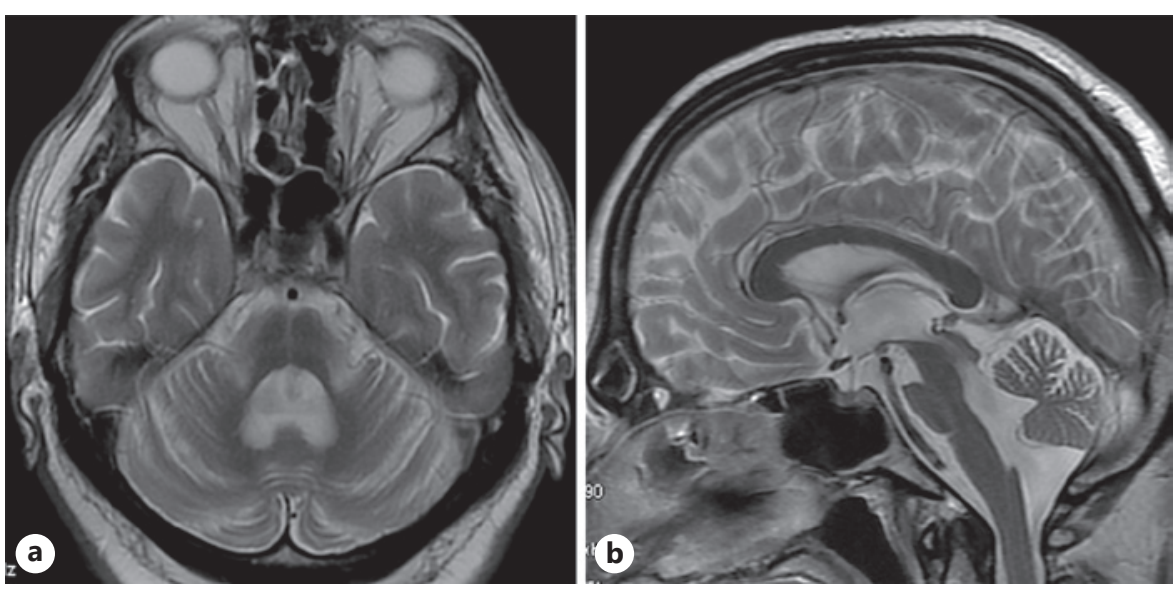

Fig. 6. a Axial T2-weighted image of a patient with MSA-C shows the typical hot cross bun sign - a cruciform hyperintensity in the central part of the atrophied pons. $\mathbf{b}$ Sagittal T2-weighted image depicts atrophy of base of pons and vermis of cerebellum, the pons seems to be "flattened." MSA-C, multiple system atrophy-cerebellar type. flect destruction of neurons and astrocytic gliosis in transverse pontocerebellar fibres with relative preservation of corticospinal tracts and tectum of pons and are the typical imaging findings of MSA-C (Fig. 6a). The appearance of sign is correlated with the advanced progression of disease $[22,23]$ as well as there is a correlation between grade of atrophy of pontine base with cerebellar vermis (Fig. 6b) and the presence of hot cross bun sign [23]. Features such as atrophy of lower brainstem, middle cerebellar peduncles, and cerebellum may occur as well $[19,24]$.

\section{Corticobasal Degeneration}

CBD is a disease with heterogeneous clinical picture, which complicates the final correct diagnosis [25]. Deposits of hyperphosphorylated microtubule associated with tau protein in the cortex (premotor, supplementary motor, and somatosensory) and in the brainstem, and basal ganglia are the fundamental microstructural alterations in CBD [26]. Due to the difficulty of making the diagnosis of CBD in vivo, occurrence of this condition is estimated only from 25 to $56 \%$ of CBD prior to autopsy [25]. Consequently, the precise assessment of CBD prevalence is not possible [27]. There are 4 phenotypes of CBD distinguished: corticobasal syndrome (CBS), frontal behavioural-spatial syndrome, nonfluent/agrammatic vari- ant (NAV) of primary progressive aphasia and PSP syndrome. Making a diagnosis of possible or probable CBD is based on combination of the clinical symptoms [23]. CBS is an asymmetric, akineto-rigid syndrome with dystonia, myoclonus and cortical dysfunction (apraxia, loss of cortical sensitivity, and alien limb phenomenon), insidious onset, and gradual progression $[25,28]$. There may be cognitive and behavioural changes early in the course of CBS $[25,26,29]$.

MRI findings are quite specific of $\mathrm{CBD}$ and can be very helpful in distinguishing CBD from the healthy brain or, more importantly, from other neurodegenerative diseases [28]. According to Albrecht et al. [28], CBD patients diagnosed clinically and all CBD subjects confirmed histopathologically have the same imaging features on MRI, which are areas of grey matter atrophy in 4 specific regions: (1) bilateral in thalamus, (2) bilateral in the posterior fronto-median cortex/posterior midcingulate cortex and premotor area/supplementary motor area, (3) in the left posterior superior frontal sulcus and middle frontal and precentral gyrus, and (4) the left posterior superior frontal sulcus and middle frontal gyrus [28]. However, regional atrophy in frontal, parietal, temporal, and occipital lobes is encountered as well [28]. Importantly, these changes occur in an asymmetrical manner. The atrophy prevails in the left hemisphere [30]. However, the right-sided features of CBD can also be found (Fig. 7) 

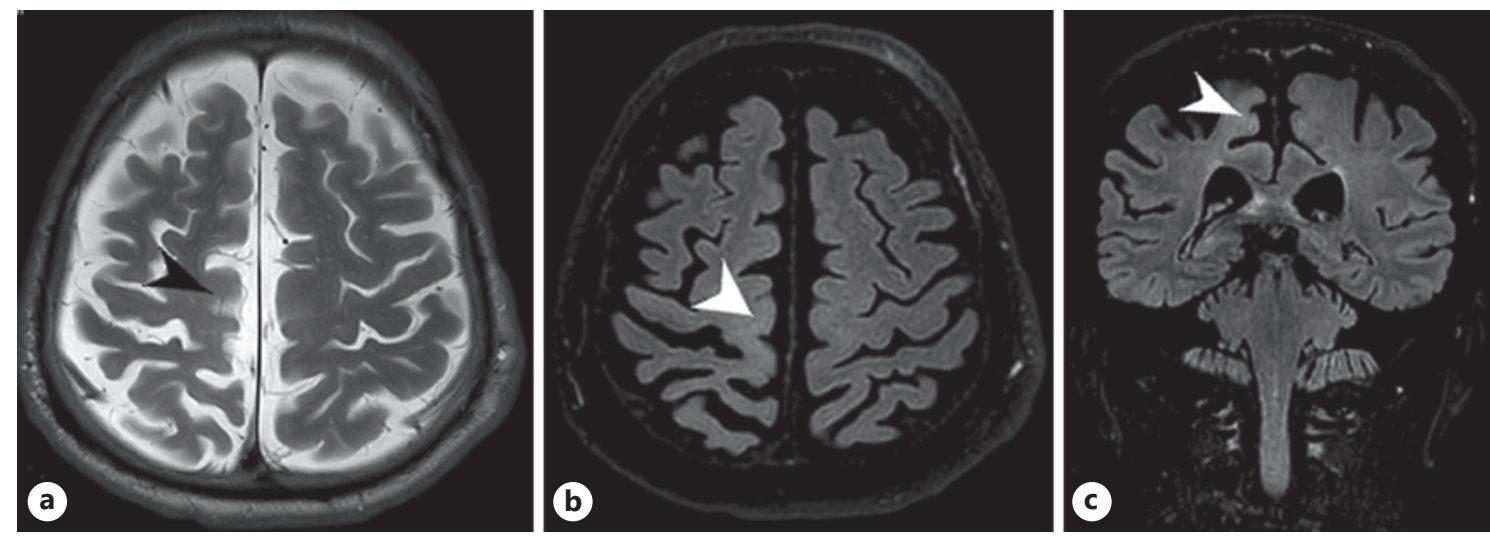

Fig. 7. A patient with CBD. Axial T2-weighted (a), axial FLAIR (b) and coronal FLAIR (c) images show asymmetric cortical atrophy in the right hemisphere. There are also subcortical hyperintense areas concomitant to the atrophied sphere (arrowheads). CBD, corticobasal degeneration.

[31]. Another characteristic features are hyperintense regions on fluid-attenuated inversion recovery and T2weighed images (T2WI) in the subcortical and periventricular white matter (WM) in areas concomitant to the hemisphere in which regional atrophy is more advanced [32].

\section{Creutzfeldt-Jakob Disease}

CJD is a prion disease with 4 subtypes. The familial CJD (fCJD) is related to mutation in PrP gene. The iatrogenic CJD (iCJD) is transmitted by growth hormone acquired from pituitary glands, corneal transplant, or dura mater grafts. The most common subtype is sporadic CJD (sCJD) which develops due to sporadic mutation or posttranslational modification of PrP gene [33]. Recently, another subgroup came in the spotlight, described in the $\mathrm{UK}$, the variant CJD (vCJD) which is a result of consumption of meat from cattle with bovine spongiform encephalopathy and differs clinically from other subtypes [34]. Incidence of CJD is estimated as $1.5-2$ cases per million per year [35]. Mean survival of sCJD is about 6 months and $>90 \%$ of affected patients die in a year from the symptom onset. The incidence is the highest in the seventh decade of life [36]. Classic sCJD is recognized based on rapidly evolving clinical symptoms such as ataxia, myoclonus, and cognitive disorders, which resulted after all in akinetic mute state leading to death usually within 1 year [36]. In the early stages of sCJD, patients may develop headache, fatigue, vertigo, sleep disorders, memory problems, behavioural changes (depression, apathy), incoor- dination, and vision loss. As the disease advances, rapidly worsening confusion, disorientation, muscle stiffness, and myoclonus can appear. Extrapyramidal symptoms like bradykinesia, dystonia, and rigidity can occur. Patients with CJD gradually lose speech, mobility and develop into a coma [34-38].

SCJD is characterized by hyperintensity of signal on T2-weighted, FLAIR, and DWI images in particular areas [37]. FLAIR and DWI sequences should be carefully evaluated in the early stage of the disease, as they ensure more reliable results $[38,39]$. The most commonly affected regions demonstrate hyperintense signal are the basal ganglia and cerebral cortex; however, the high-signal intensity may also appear in both thalami (Fig. 8a) [40]. Occasionally, signs such as hockey stick sign or pulvinar sign are observed in the thalami on T2-weighted or/and in FLAIR images as well as on DWI (Fig. 8a) [41]. Combination of the abnormal signal intensity in the caudate nucleus and putamen or 2 out of 3 cerebral cortical regions (parietal-temporal-occipital) on DWI images can be used as a test to approve possible CJD in a conjunction with the clinical symptoms (Fig. 8a, b). The imaging findings with the Radermecker-type complexes in EEG and detection of the 14.3.3 protein in the cerebrospinal fluid give $98 \%$ sensitivity and $70.8 \%$ specificity, compared to previous criteria, using only EEG results and the presence of the 14.3.3 protein, respectively 92.2 and $71.2 \%$ [38]. It should be stressed that the most important sequence in the early CJD diagnosis is DWI. Hyperintensities on the trace images of DWI in the basal ganglia and thalami or/and in the cortex with corresponding hypointensities on ADC map (i.e., restricted diffusion) are very suggestive of the 
Fig. 8. Axial DWI showing early restriction of diffusion in basal ganglia (a, black, white, and grey arrows), thalami (a, hockey stick/ pulvinar sign - grey arrow), and cortex (b, circle). DWI, diffusion-weighted imaging.
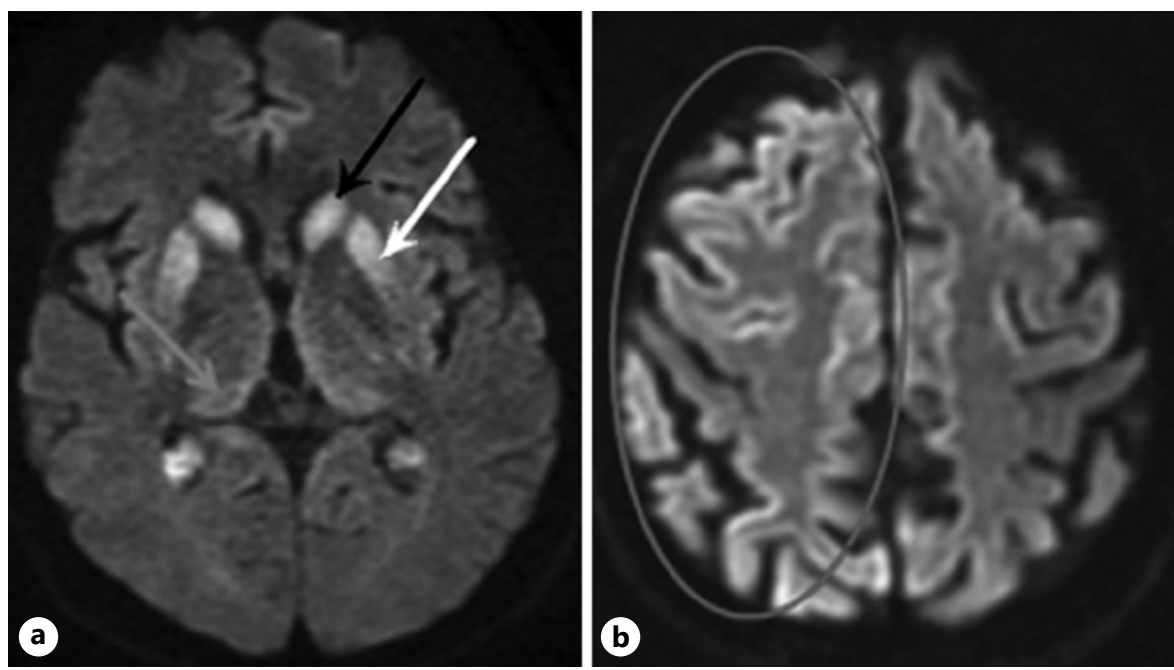

Fig. 9. T2-weighted images in axial (a) and coronal (b) plane in a patient with HD atrophy of caudate nuclei (black arrows) with secondary enlargement of frontal horns of lateral ventricles. Furthermore, there is visible hyperintensity of putamina (white arrow) and caudate nuclei (black arrow). Note the typical shape of the widened frontal horns. HD, Huntington's disease.
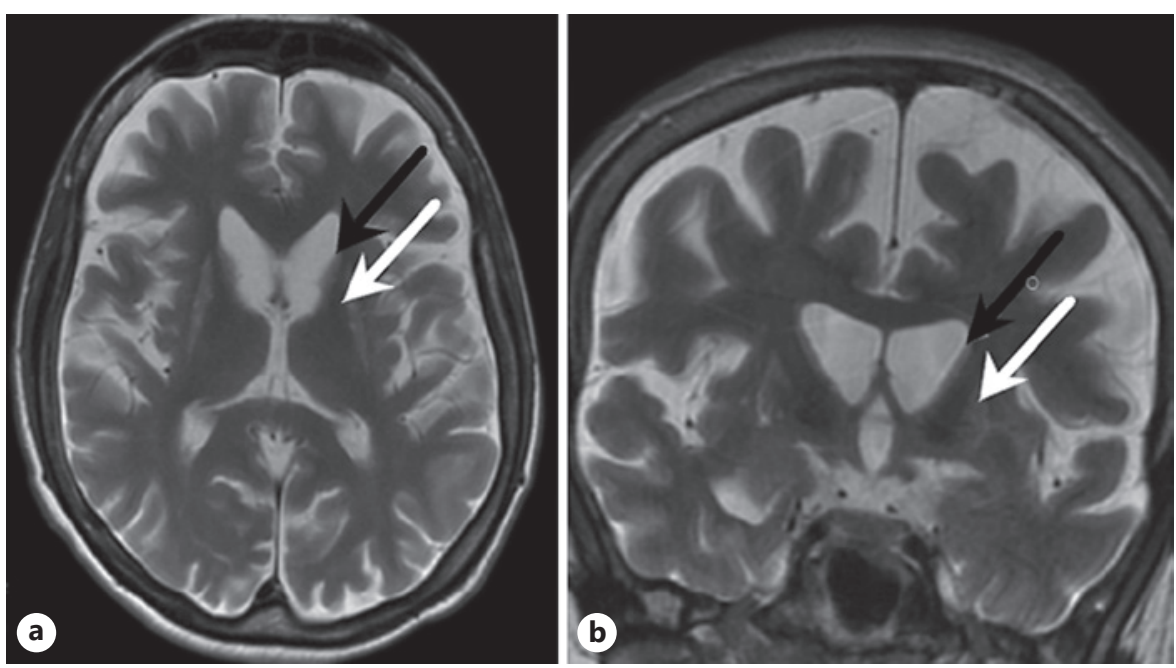

diagnosis [39]. Restriction of diffusion is not seen in nonprion causes of rapidly progressive dementia, which allows to diagnose SCJD in the early stage. However, note that hyperintensities on FLAIR can also indicate other causes of dementia than SCJD [42].

\section{Huntington's Disease}

HD is caused by multiplied CAG triplet in huntingtin (HTT) gene leading to production of the defective HTT protein. The incorrect HTT protein seems to disrupt balance in many tissues, primarily in the central nervous system [43]. Prevalence is dependent on region and varies from 0.11 per 100,000 in Asia to 17.27 per 100,000 in Canada [44]. The clinical symptoms of HD are divided into 3 groups: motor (chorea, dystonia, ataxia, motor impersis- tence, atypical parkinsonism, and eye movement abnormalities), cognitive, and psychiatric syndromes. Chorea affecting distal extremities and facial muscles as well as deficit in the postural control with recurrent falls are characteristic signs of the group of motor symptoms. The cognitive disorders can be the first sign of $\mathrm{HD}$ and may occur as mild cognitive impairment even 15 years before motor symptoms appear. Cognitive deficits involve executive functions (planning, multitasking, word generation, processing speed, and memory recall). In the advanced stage of the disease, increasing cognitive dysfunction results in a dementia syndrome, in which disrupted functional abilities and deteriorating dominate over memory deficit. The psychiatric symptoms, such as depression, psychosis, anxiety, antisocial behaviours, aggression, irritability, disinhibition, apathy, and misidentification syndrome, place patients in a higher risk group 
Fig. 10. a T2-weighted image demonstrating giant panda sign in a patient with WD. High signal in tegmentum and normal signal of red nuclei forms "eyes" (arrowheads), high signal of lateral part of pars reticulata of substantia nigra forms "ears" and hypointensive superior colliculus forms "mouth." b Axial T1-weighted image in the same patient showing typical high-signal intensity of globi pallidi. WD, Wilson disease.
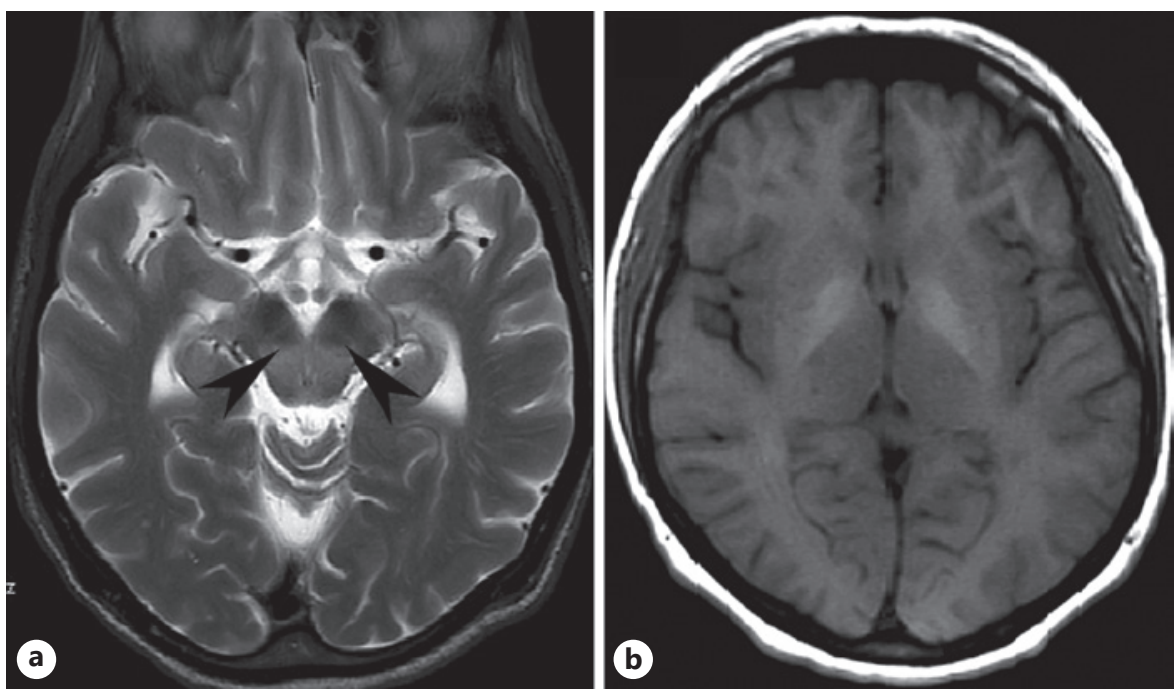

for the suicidal intention. Patients also often lack insight into their motor and cognitive impairment [43-45].

Atrophy of striatum both caudate nuclei and putamen with secondary enlargement of lateral ventricles is the typical feature on structural MRI (Fig. 9). The enlarged frontal horns of lateral ventricles demonstrate a characteristic box-like configuration [45]. The severity of the disease correlates with the atrophy of striatum, especially symptoms connected with the capacity of function, executive function, and speed of processes and also with memory deficits [46]. Moreover, the striatum volume is negatively correlated with CAG triplets repeat [46]. Carriers of mutated HTT gene have striatal volume reduction even 23 years prior to the motor symptoms occurrence [47]. Besides the striatum, there are multiple structures affected by atrophy. Extra-striatal atrophy in clinical HD occurs in the thalamus, globus pallidus, insula, hippocampus, amygdala, cerebellum, and hypothalamus. In preclinical HD, atrophy can be found in the globus pallidus, thalamus in addition to the striatal abnormalities (Fig. 9) [46].

\section{Wilson Disease}

WD leads to various neurologic and psychiatric disorders. It is caused by accumulating copper, primarily in the liver. Since the copper excretion with bile is impossible, copper stores in other body tissues, including the brain. It is autosomal recessive disorder caused by gene ATP7B mutation and production of the defective protein ATP7B, which is responsible for copper transport. When liver concentration ability exhausts, copper accumulates in other organs, causing additionally the ophthalmic, hematologic, musculoskeletal, myocardial, renal, and dermatologic symptoms [48]. The prevalence of mutant gene carriers is estimated to be 142 per million [49]. Significant differences in the phenotype can occur in the carriers of the same type mutation [49]. The onset of the neurologic symptoms ranges between 6 and 72 years. The classic neurologic representation of this disease is coarse proximal tremor of upper extremities, like a wing-beating bird [48]. The psychiatric symptoms involve a variety of disturbances from subtle personality changes to severe psychosis syndromes. Mild cognitive impairment is more common than dementia in WD, although dementia can develop in the advanced stages [48]. The differential diagnosis for WD should be considered for every patient with the psychiatric or neurologic symptoms with liver dysfunction, particularly in young patients [48].

Accumulation of copper in the brain tissue is heterogeneous across the brain. The highest copper concentration is observed in the locus coeruleus, followed by the substantia nigra, globus pallidus, and cerebral cortex. High concentration of copper is also recorded in the subcortical white matter (WM) and brainstem [50, 51]. Almost every patient with neurological symptoms reveals pathological changes on MRI examination. Moreover, almost $20 \%$ of presymptomatic cases present the brain abnormalities. Besides brain atrophy, there are typical symmetric hyperintensities on T2-weighted images in the basal ganglia, particularly in putamina, caudate nuclei, globi pallidi, also in thalami and pons. In more advanced cases, hypointense areas on T1-weighted images in the 

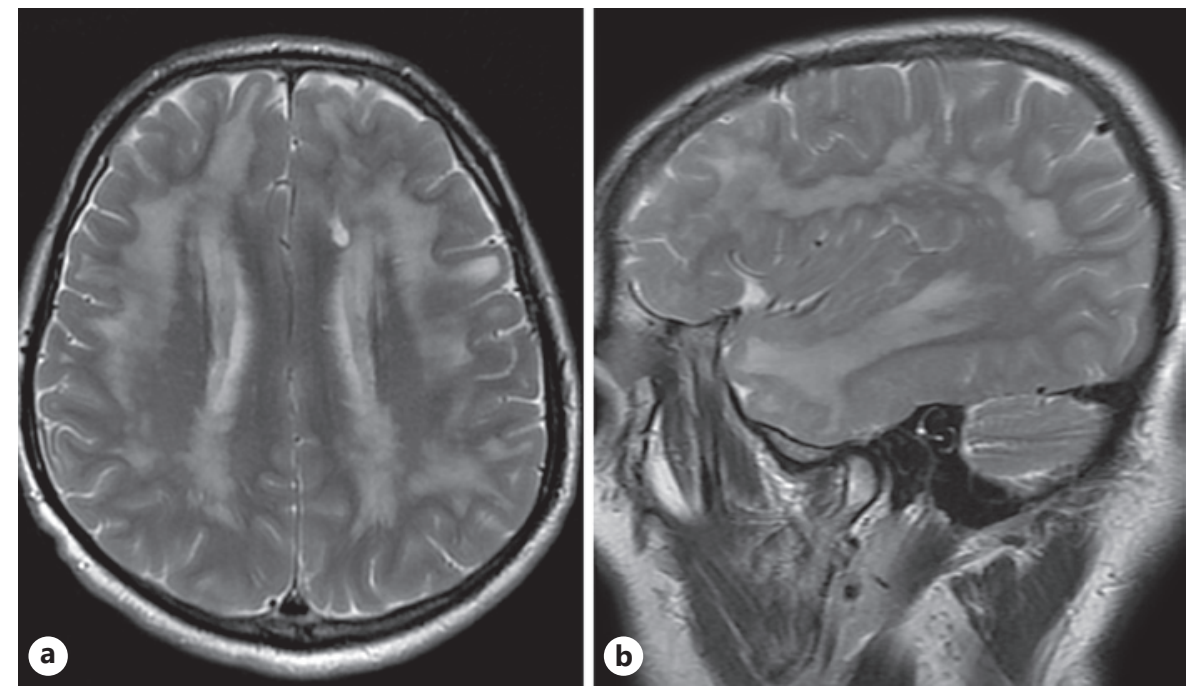

Fig. 11. a Axial T2-weighted image in a 35-year-old patient with CADASIL. Bilateral hyperintense regions in frontal and parietal lobes are visible. b Sagittal T2-weighted image in the same patient shows very characteristic hyperintense areas in the anterior part of the temporal lobe. CADASIL, cerebral autosomal dominant arteriopathy with subcortical infarcts and leucoencephalopathy.

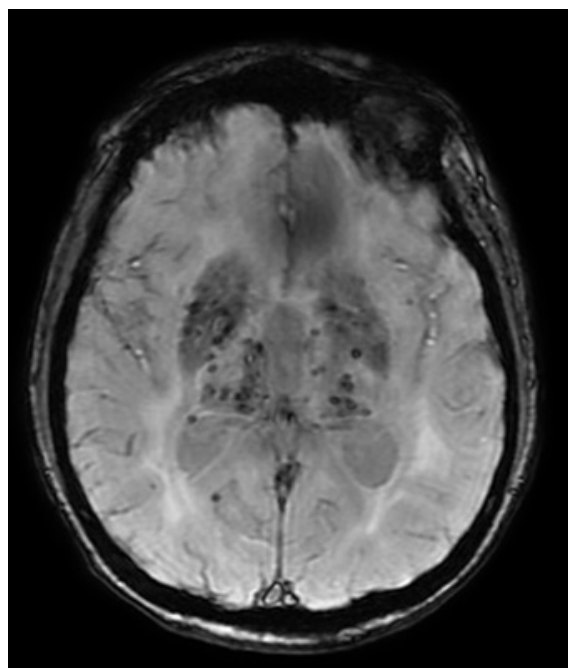

Fig. 12. Axial SWI presenting multiple "black dots" in the basal ganglia in a patient with CADASIL. SWI, susceptibilityweighted imaging; CADASIL, cerebral autosomal dominant arteriopathy with subcortical infarcts and leucoencephalopathy. midbrain, cerebellum, WM, and corticospinal tracts may be seen $[51,52]$. The finding called "giant panda sign" which is seen in the midbrain is a highly suggestive feature of WD (Fig. 10a). On T2-weighted images, high signal in tegmentum and normal signal of the red nuclei forms "eyes", high signal of lateral part of pars reticulata of substantia nigra forms "ears" and hypointense superior colliculus forms "mouth" [52]. There is also miniature panda sign or panda cub sign which occur in the pons with hypointensity of the central tegmental tracts and hyperintensity of the aqueductal opening of the 4 th ventricle [52]. The coincidence of this 2 sings is called the double panda sign. T1-weighted hyperintensities in the basal ganglia are typical, which are caused by the accumulation of copper (Fig. 10b) [53].

\section{Cerebral Autosomal Dominant Arteriopathy with Subcortical Infarcts and Leukoencephalopathy}

CADASIL is a rare monogenic cerebral small vessel disease. The clinical symptoms are widely variable and usually start in early adulthood. They include migraines with aura, recurrent subcortical strokes, gradual cognitive dysfunction or pure psychiatric manifestations, and acute encephalopathy [54]. There is a higher incidence of depression and suicidal incidents. The neurologic symptoms are more common than psychiatric ones and correspond to eloquent brain areas [55]. The prevalence varies between 2 and 5 in 100,000 [55].

On MRI, there are diffuse symmetrical hyperintense areas in the WM on T2-weighted images. These lesions are located in the anterior temporal lobes, external capsules, superior frontal gyrus, basal nuclei, and thalamus bilaterally $[55,56]$. The presence of the hyperintense areas in the anterior parts of temporal lobes are the typical features of CADASIL and differentiate it from other entities (Fig. 11a, b) [55, 56]. T2-weighted hyperintense lesions in the corpus callosum are encountered in CADASIL as well as in the multiple sclerosis, while the optic nerves and the spinal cord are not involved in the spectrum of CADASIL [55]. Sporadic subcortical arteriosclerotic encephalopathy has similar manifestation on MRI, but hyperintense alterations in the temporal lobes clearly indicate CADASIL [56]. Such lesions can also point to diagnosis of leukoaraiosis, although these are not so extensive and usually appear around the ventricles [57]. Other features of CADASIL are the multiple small round low signal intensity areas called as "black dots" on the SWI (Fig. 12). These changes occur due to microhaemorrhages and significantly increased iron accumulation in the basal ganglia [58]. 


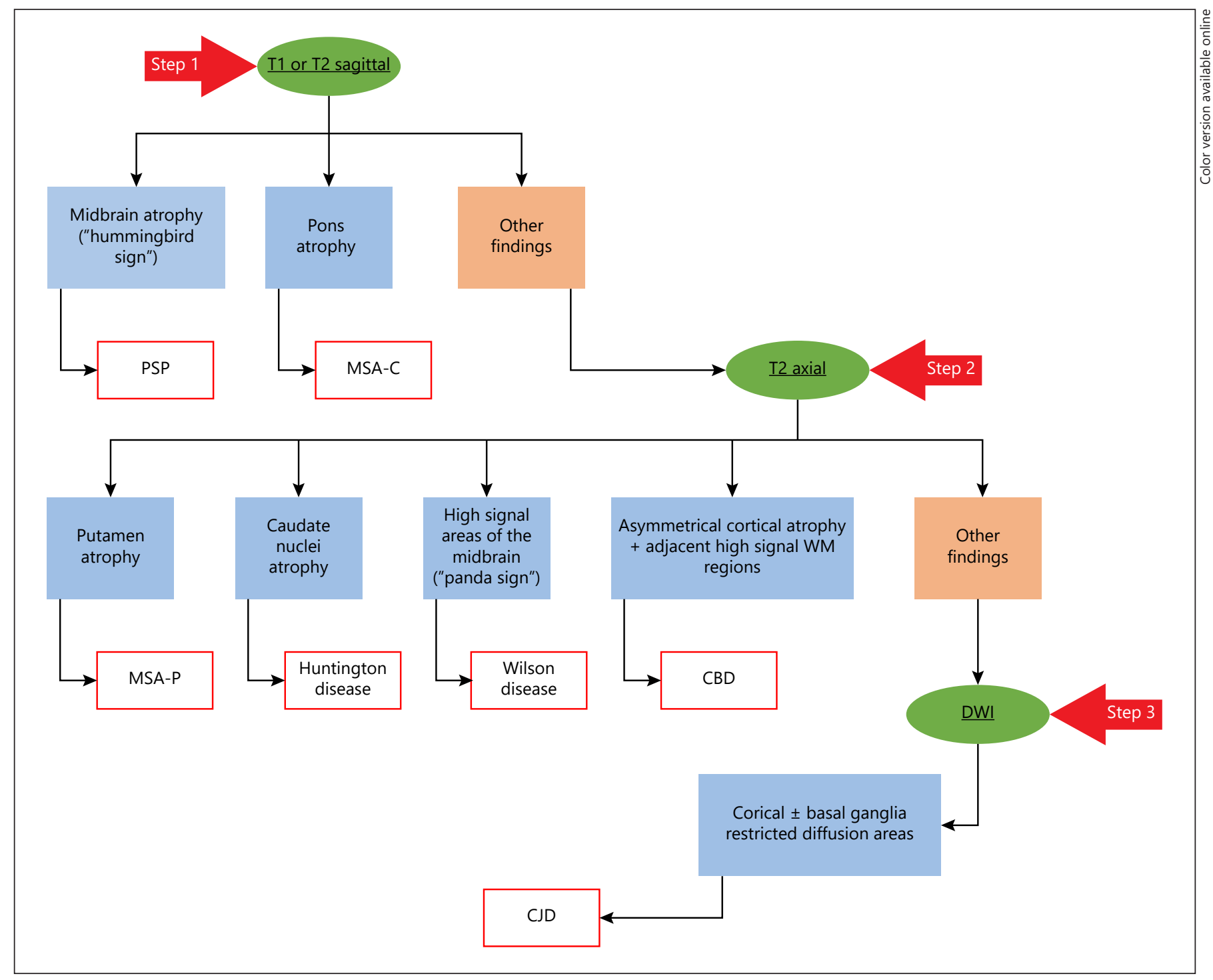

Fig. 13. A practical approach to imaging findings on conventional MRI in suspected neurodegenerative disease.

\section{A Practical Approach to MRI Findings}

In case of a suspected neurodegenerative disease in our department the following MRI protocol has been applied: sagittal, axial and coronal T2-weighted images (T2WI), T1 3D volume (T1WI), 3D fluid-attenuated inversion recovery (FLAIR), DWI (diffusion-weighted imaging), and SWI images.

To provide a practical approach to the final diagnosis, we recommend using 3 simple steps helpful in the diagnosis-making process (Fig. 13). Firstly, look at the sagittal T2-weighted images to assess the atrophy of the midbrain or pons. No signs of prominent atrophy may exclude the diagnosis of PSP and MSA-C. As the second step, assess the axial T2-weighted images for putamen atrophy (MSA$\mathrm{P})$, asymmetrical cortical atrophy with adjacent high-signal WM areas (CBD), caudate nuclei atrophy (HD), or hyperintense regions of the midbrain showing the "Panda sign" (WD). Finally, as the 3rd step, consider any areas of restricted diffusion on DWI images. High signal on DWI within cortex and/or basal ganglia region is highly suggestive of the early stage of CJD. The typical features suggestive of the particular disease are presented in the table (Table 1). 
Table 1. Shows typical features of selected neurodegenerative diseases

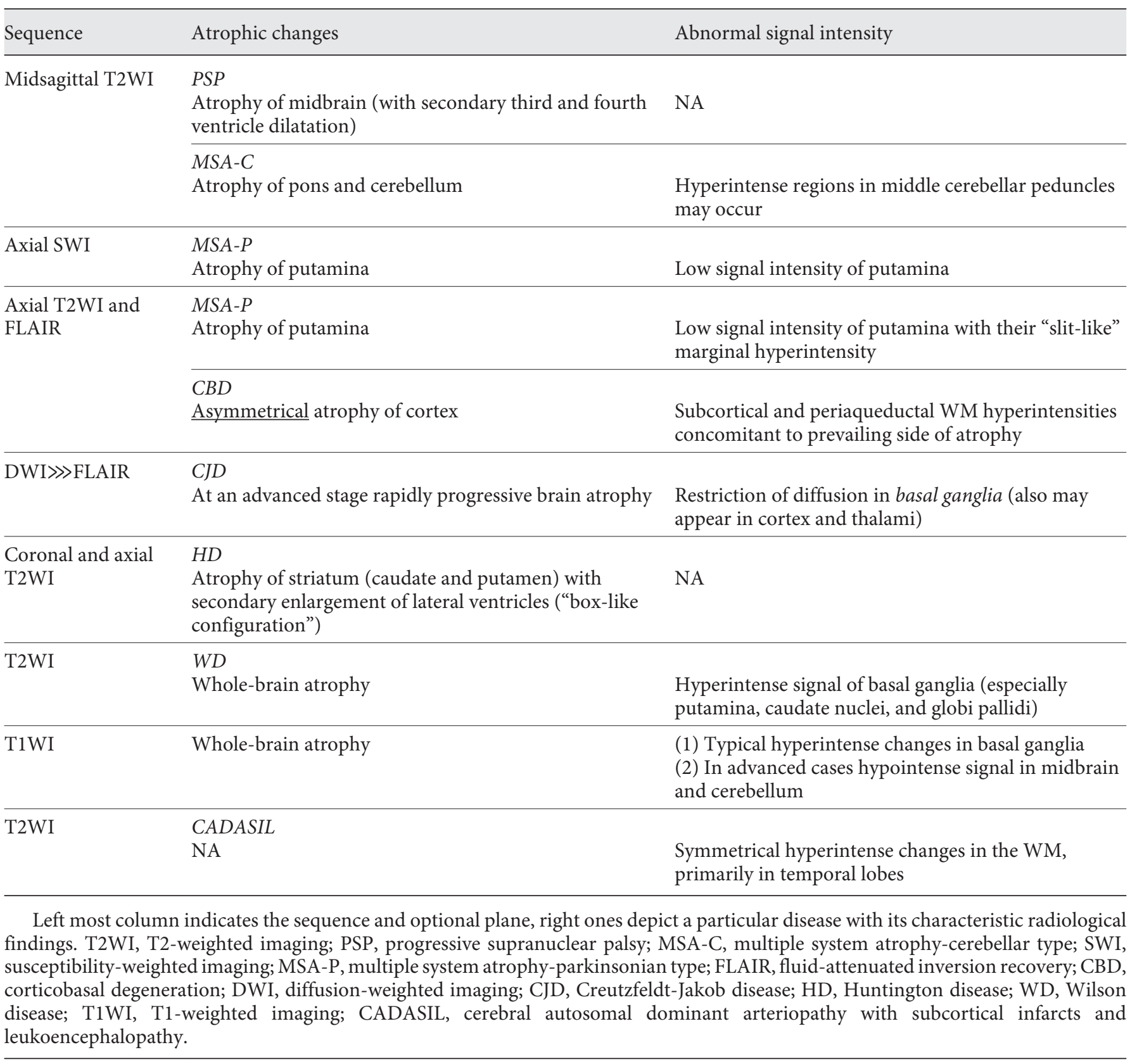

\section{Conclusions}

Neurodegenerative diseases are a huge clinical and diagnostic challenge. Despite the significant development in this field in neurology and strictly defined clinical diagnostic criteria, correct diagnosis is most often possible only in correlation with the neuroimaging features. Often, a longer neurological and radiological follow-up is necessary to make a final accurate diagnosis. In our opinion,
DWI as well as SWI techniques are mandatory sequences which should be included in the routine MRI protocol dedicated to the assessment of neurodegenerative disorders.

\section{Conflict of Interest Statement}

The authors have no conflicts of interest to declare. 


\section{Funding Sources}

The authors did not receive any funding.
A.Z.G., M.J.S., A.Z., M.W.P., and J.B. have substantially revised the manuscript. M.S., M.W.P., and J.B. have made essential contribution to the major revision of the manuscript. All authors read and approved the final manuscript.

\section{Author Contributions}

A.Z.G. has made substantial contribution to the conception. A.Z.G., J.B., and A.Z. have analysed and interpreted the patient data, and M.S. has written a manuscript draft and created graphs.

\section{Figures Source}

Department of General and Interventional Radiology and Neuroradiology, Wroclaw Medical University.

\section{References}

1 Haller S, Garibotto V, Barkhof F. Neurodegenerative disorders: classification and imaging strategy. Clin Neuroradiol. 2019;1251-75.

2 Potashkin JA, Santiago JA, Ravina BM, Watts A, Leontovich AA. Biosignatures for Parkinson's disease and atypical parkinsonian disorders patients. PLoS One. 2012;7(8):e43595.

3 Wood J, Mirra S, Pollock N, Binder L. Neurofibrillary tangles of Alzheimer disease share antigenic determinants with the axonal microtubule-associated protein tau (tau). Proc Natl Acad Sci U S A. 1986 Jun;83(11):4040-3.

4 Ruberg M, Javoy-Agid F, Hirsch E, Scatton B, Lheureux R, Hauw JJ, et al. Dopaminergic and cholinergic lesions in progressive supranuclear palsy. Ann Neurol. 1985;18(5):523.

5 Schrag A, Ben-Shlomo Y, Quinn NP. Prevalence of progressive supranuclear palsy and multiple system atrophy: a cross-sectional study. Lancet. 1999;354(9192):1771.

6 Nath U, Ben-Shlomo Y, Thomson RG, Morris HR, Wood NW, Lees AJ, et al. The prevalence of progressive supranuclear palsy (SteeleRichardson-Olszewski syndrome) in the UK. Brain. 2001 Jul;124(Pt 7):1438-49.

7 Golbe LI. Progressive supranuclear palsy. Semin Neurol. 2014;214(34):151-9.

8 Stamelou M, Knake S, Oertel WH, Höglinger GU. Magnetic resonance imaging in progressive supranuclear palsy. J Neurol. 2011; 258(4):549.

9 Adachi M, Kawanami T, Ohshima H, Sugai Y, Hosoya T. Morning glory sign: a particular MR finding in progressive supranuclear palsy. Magn Reson Med Sci. 2004;3(3):125.

10 Mori H, Aoki S, Ohtomo K. Morning glory sign is not prevalent in progressive supranuclear palsy. Magn Reson Med Sci. 2004;3(4): 215-7.

11 Mori H, Aoki S, Ohtomo K. The "morning glory sign" may lead to false impression according to slice angle. Magn Reson Med Sci. 2007;6(3):183-5.

12 Oba H, Yagishita A, Terada H, Barkovich AJ, Kutomi K, Yamauchi T, et al. New and reliable MRI diagnosis for progressive supranuclear palsy. Neurology. 2005;64(12):2050.
13 Möller L, Kassubek J, Südmeyer M, Hilker R, Hattingen E, Egger K, et al. Manual MRI morphometry in Parkinsonian syndromes. Mov Disord. 2017;32(5):778.

14 Whitwell JL. Tau imaging in Parkinsonism: what have we learned so far? Mov Disord Clin Pract. 2018;5(2):118-30.

15 Laurens B, Vergnet S, Lopez MC, FoubertSamier A, Tison F, Fernagut PO, et al. Multiple system atrophy: state of the art. Curr Neurol Neurosci Rep. 2017;17(5):41.

16 Fanciulli A, Wenning GK. Multiple-system atrophy. N Engl J Med. 2015;372(3):249-63.

17 Bower JH, Maraganore DM, McDonnell SK, Rocca WA. Incidence of progressive supranuclear palsy and multiple system atrophy in Olmsted County, Minnesota, 1976 to 1990. Neurology. 1997;49(5):1284.

18 Gilman S, Wenning GK, Low PA, Brooks DJ, Mathias CJ, Trojanowski JQ, et al. Second consensus statement on the diagnosis of multiple system atrophy. Neurology. 2008;71(9):670.

19 Seppi K, Schocke MF, Wenning GK, Poewe W. How to diagnose MSA early: the role of magnetic resonance imaging. J Neural Transm. 2005;112(12):1625.

20 Wang Y, Butros SR, Shuai X, Dai Y, Chen C, Liu M, et al. Different iron-deposition patterns of multiple system atrophy with predominant parkinsonism and idiopathetic parkinson diseases demonstrated by phasecorrected susceptibility-weighted imaging. AJNR Am J Neuroradiol. 2012;33(2):266.

21 Schwarz J, Weis S, Kraft E, Tatsch K, Bandmann O, Mehraein P, et al. Signal changes on MRI and increases in reactive microgliosis, astrogliosis, and iron in the putamen of two patients with multiple system atrophy. J Neurol Neurosurg Psychiatry. 1996;60(1):98.

22 Kasahara S, Miki Y, Kanagaki M, Kondo T, Yamamoto A, Morimoto E, et al. "Hot cross bun" sign in multiple system atrophy with predominant cerebellar ataxia: a comparison between proton density-weighted imaging and T2-weighted imaging. Eur J Radiol. 2012 Oct;81(10):2848-52.

23 Abe K, Hikita T, Yokoe M, Mihara M, Sakoda $\mathrm{S}$. The "cross" signs in patients with multiple system atrophy: a quantitative study. J Neuroimaging. 2006;16(1):73.
24 Kadodwala VH, Hadjivassiliou M, Currie S, Skipper N, Hoggard N. Is 1H-MR spectroscopy useful as a diagnostic aid in MSA-C? Cerebellum Ataxias. 2019 Jul 5;6:7.

25 Armstrong MJ, Litvan I, Lang AE, Bak TH, Bhatia KP, Borroni B, et al. Criteria for the diagnosis of corticobasal degeneration. Neurology. 2013;80(5):496-503.

26 Ling H, Kovacs GG, Vonsattel JP, Davey K, Mok KY, Hardy J, et al. Astrogliopathy predominates the earliest stage of corticobasal degeneration pathology. Brain. 2016;139(Pt 12):3237.

27 Dickson DW. Neuropathologic differentiation of progressive supranuclear palsy and corticobasal degeneration. J Neurol. 1999 Sep; 246(Suppl 2):116-5.

28 Albrecht F, Bisenius S, Morales Schaack R, Neumann J, Schroeter ML. Disentangling the neural correlates of corticobasal syndrome and corticobasal degeneration with systematic and quantitative ALE meta-analyses. NPJ Parkinsons Dis. 2017;3:12.

29 Singh TD, Duffy JR, Strand EA, Machulda MM, Whitwell JL, Josephs KA. Neuropsychiatric symptoms in primary progressive aphasia and apraxia of speech. Dement Geriatr Cogn Disord. 2015;39(3-4):228.

30 Gil-Navarro S, Lladó A, Rami L, Castellví M, Bosch B, Bargalló N, et al. Neuroimaging and biochemical markers in the three variants of primary progressive aphasia. Dement Geriatr Cogn Disord. 2013;35(1-2): 106.

31 Whitwell JL, Jack CR, Boeve BF, Parisi JE, Ahlskog JE, Drubach DA, et al. Imaging correlates of pathology in corticobasal syndrome. Neurology. 2010;75(21):1879.

32 Josephs KA, Tang-Wai DF, Edland SD, Knopman DS, Dickson DW, Parisi JE, et al. Correlation between antemortem magnetic resonance imaging findings and pathologically confirmed corticobasal degeneration. Arch Neurol. 2004;61(12): 1881.

33 Armstrong RA. Laminar distribution of the pathological changes in sporadic and variant Creutzfeldt-Jakob disease. Patholog Res Int. 2010 Dec 16;2011:236346.
Neuroimaging of the Rare

Neurodegenerative Diseases
Dement Geriatr Cogn Disord 2020;49:544-556 DOI: $10.1159 / 000512543$ 
34 Will RG, Ironside JW, Zeidler M, Cousens SN, Estibeiro K, Alperovitch A, et al. A new variant of Creutzfeldt-Jakob disease in the UK. Lancet. 1996;347(9006):921.

35 Mackenzie G, Will R. Creutzfeldt-Jakob disease: recent developments. F1000Res. 2017 Nov 27;6:2053.

36 Ladogana A, Puopolo M, Croes EA, Budka H, Jarius C, Collins S, et al. Mortality from Creutzfeldt-Jakob disease and related disorders in Europe, Australia, and Canada. Neurology. 2005;64(9):1586.

37 Paradowski M, Bladowska J, Paradowski B. MRI in a patient with sporadic CreutzfeldtJakob disease with over 72 months survival. Neurol India. 2017;65(6):1441-2.

38 Zerr I, Kallenberg K, Summers DM, Romero C, Taratuto A, Heinemann U, et al. Updated clinical diagnostic criteria for sporadic Creutzfeldt-Jakob disease. Brain. 2009;132(Pt 10):2659.

39 Eisenmenger L, Porter MC, Carswell CJ, Thompson A, Mead S, Rudge P, et al. Evolution of diffusion-weighted magnetic resonance imaging signal abnormality in sporadic Creutzfeldt-Jakob disease, with histopathological correlation. JAMA Neurol. 2016; 73(1):76.

40 Meissner B, Kallenberg K, Sanchez-Juan P, Collie D, Summers DM, Almonti S, et al. MRI lesion profiles in sporadic Creutzfeldt-Jakob disease. Neurology. 2009;72(23):1994.

41 Krasnianski A, Schulz-Schaeffer WJ, Kallenberg K, Meissner B, Collie DA, Roeber S, et al. Clinical findings and diagnostic tests in the MV2 subtype of sporadic CJD. Brain. 2006; 129(Pt 9):2288.
42 Vitali P, MacCagnano E, Caverzasi E, Henry RG, Haman A, Torres-Chae C, et al. Diffusion-weighted MRI hyperintensity patterns differentiate CJD from other rapid dementias. Neurology. 2011;76(20):1711.

$43 \mathrm{Ha}$ AD, Fung VS. Huntington's disease. Curr Opin Neurol. 2012;25(4):491.

44 Rawlins MD, Wexler NS, Wexler AR, Tabrizi SJ, Douglas I, Evans SJW, et al. The prevalence of Huntington's disease. Neuroepidemiology. 2016;46(2):144-53.

45 Rishi PM, Binston T, Ram Basti S. Huntington's disease: an easily overlooked entity. 2013.

46 Bohanna I, Georgiou-Karistianis N, Hannan AJ, Egan GF. Magnetic resonance imaging as an approach towards identifying neuropathological biomarkers for Huntington's disease. Brain Res Rev. 2008;58(1):209.

47 Klöppel S, Henley SM, Hobbs NZ, Wolf RC, Kassubek J, Tabrizi SJ, et al. Magnetic resonance imaging of Huntington's disease: preparing for clinical trials. Neuroscience. 2009 Nov 24;164(1):205-19.

48 Pfeiffer RF. Wilson disease. Continuum. 2016;22(August):1246-61.

49 Lo C, Bandmann O. Epidemiology and introduction to the clinical presentation of Wilson disease. Handb Clin Neurol. 2017;142:7.

50 Scheiber IF, Brůha R, Dušek P. Pathogenesis of Wilson disease. Handb Clin Neurol. 2017; 142:43.

51 Yu XE, Gao S, Yang RM, Han YZ. MR imaging of the brain in neurologic Wilson disease. Am J Neuroradiol. 2019 Jan;40(1):178-83.
52 Członkowska A, Litwin T, Chabik G. Wilson disease: neurologic features. Handb Clin Neurol. 2017;142:101-19.

53 Zimny A, Zińska L, Bladowska J, Neska-Matuszewska M, Sąsiadek M. Intracranial lesions with high signal intensity on T1-weighted MR images: review of pathologies. Pol J Radiol. 2013;78(4):36.

54 Markiewicz R, Masiak J. Evaluation of cognitive deficits in schizophrenia using event-related potentials and rehabilitation influences using EEG Biofeedback in patients diagnosed with schizophreni. Psychiatr Pol. 2019 Dec 31;53(6):1261-73.

55 Di Donato I, Bianchi S, De Stefano N, Dichgans M, Dotti MT, Duering M, et al. Cerebral autosomal dominant arteriopathy with subcortical infarcts and leukoencephalopathy (CADASIL) as a model of small vessel disease: update on clinical, diagnostic, and management aspects. BMC Med. 2017; 15(1):41.

56 Auer DP, Pütz B, Gössl C, Elbel G, Gasser T, Dichgans M. Differential lesion patterns in CADASIL and sporadic subcortical arteriosclerotic encephalopathy: MR imaging study with statistical parametric group comparison. Radiology. 2001;218(2):443.

57 Marek M, Horyniecki M, Frączek M, Kluczewska E. Leukoaraiosis: new concepts and modern imaging. pjr. 2018;83:76-81.

58 Mittal S, Wu Z, Neelavalli J, Haacke EM. Susceptibility-weighted imaging: technical aspects and clinical applications, part 2. AJNR Am J Neuroradiol. 2009;30(2):232. 